\title{
Biblioteca digital: bibliografia das principais fontes de informação
}

\author{
Murilo Bastos da Cunha \\ Doutor pela University of Michigan, Ann Arbor, Estados Unidos. \\ Doutorado em Library and Information Science. University of \\ Michigan, Estados Unidos. \\ E-mail:murilobc@unb.br
}

\section{Resumo}

Bibliografia internacional anotada sobre as principais fontes de informação lançadas a partir de 2000, relacionadas à biblioteca digital. Os tópicos cobertos são as bibliografias mais recentes, os livros e manuais, os periódicos especializados, os eventos específicos, as listas de discussão, os grupos e centros de pesquisa, os cursos e treinamentos e as principais organizações.

\section{Palavras-chave}

Bibliografia. Biblioteca digital.

\section{Digital library: a bibliography of major information sources}

\begin{abstract}
International annotated bibliography about the major information sources related to digital library. The topics covered are: most recent bibliographies, books and manuals, specialized journals, specialized conferences, discussion lists, research groups and centers, training courses and major organizations.
\end{abstract}

\section{Keywords}

Bibliography. Digital library.

\section{INTRODUÇÃO}

O assunto biblioteca digital teve, a partir de meados dos anos 1990, enorme crescimento. Isto foi consubstanciado, entre outros motivos, pelo surgimento de livros e eventos profissionais específicos, bem como pela publicação de centenas de artigos. Tais fatos demonstram, por conseguinte, a necessidade de uma bibliografia na qual constassem as principais fontes de informação que pudessem delinear os contornos dessa dinâmica área. Este é, portanto, o objetivo deste trabalho: incluir, de forma anotada, os principais documentos sobre biblioteca digital, disponibilizados nas línguas mais acessíveis aos leitores brasileiros.

Este artigo destina-se aos estudantes e profissionais da biblioteconomia, arquivologia e demais áreas da ciência da informação. Eles podem encontrar, em um só documento, referências básicas relacionadas com os principais problemas e soluções envolvidos na complexa construção de uma biblioteca digital.

\section{AS PRINCIPAIS FONTES DE INFORMAÇÃO}

Esta bibliografia abordou as principais fontes de informação lançadas a partir de 2000, necessárias ao estudo global das bibliotecas digitais. Foram incluídos oito tipos de fontes, a saber: as bibliografias mais recentes, os livros e manuais, os periódicos especializados, os eventos específicos, as listas de discussão, os grupos e centros de pesquisa, os cursos e treinamentos, as principais organizações da área. Dentro de cada tópico, as entradas dos itens seguem o arranjo alfabético.

Em cada um desses tipos de fontes de informação foram comentados os documentos básicos, brasileiros ou estrangeiros, em ordem alfabética dos autores e/ou instituições. Tanto quanto possível, foram incluídos os endereços eletrônicos na internet.

\section{Bibliografias}

As bibliografias sobre bibliotecas digitais têm crescido bastante na internet; a seguir estão citadas as mais recentes.

Ci. Inf., Brasília, DF, v. 39 n. 1, p.88-107, jan./abr., 2010 
BAILEY JUNIOR, Charles W. Scholarly electronic publishing bibliography: 2008 annual edition. Houston: Digital Scholarship, 2009, 268 p. Disponível em: www.digital-scholarship.org/sepb/sepb.html. Bibliografia que inclui mais de três mil itens com artigos de periódicos, livros, documentos e fontes digitais relacionadas com publicações acadêmicas eletrônicas. Abrange noves áreas: problemas econômicos, livros e textos eletrônicos, periódicos eletrônicos, obras gerais, problemas legais, problemas bibliotecários (catalogação, metadados, bibliotecas digitais, preservação), novos modelos de publicação, gestão dos direitos digitais, repositórios e arquivos abertos.

DAY, Michael. Preservation of electronic information: a bibliography. Bath: UKOLN, 2000. Disponível em: $<$ http://homes.ukoln.ac.uk/ lismd/preservation. html>. Bibliografia internacional sobre preservação da informação digital com ênfase na língua inglesa.

INFORMATION Architecture Library. Disponível em: <http://iainstitute.org/library/>. Biblioteca mantida pelo Information Architecture Institute, que provê acesso a artigos, livros, blogs e páginas da Web relacionados à arquitetura da informação.

INTERNATIONAL FEDEREATION OF LIBRARY ASSOCIATIONS AND INSTITUTIONS . Digital libraries: resources and projects. Disponível em: < http://www.ifla.org/II/ diglib.htm $>$. Bibliografia de cunho internacional sobre coleções, periódicos, conferências, organizações e projetos de bibliotecas digitais.

LOPATIN, Laurie. Library digitization projects, issues and guidelines: a survey of the literature. Library Hi Tech, v. 24, n. 2, p. 273-289, 2006. Bibliografia seletiva com comentários sobre a literatura publicada entre 2000 e 2005, relacionada aos projetos, problemas e guias relacionados com digitalização.

STANFORD DIGITAL LIBRARIES PROJECT (THE). Annotated bibliography of digital library related sources, 2006. Disponível em: <http://liinwww.ira.
uka.de/bibliography/Misc/digital.library.html> . Bibliografia com cerca de 1.400 referências, com um sistema que permite busca avançada.

UNIVERSITY of California. Berkeley digital library sunsite. Disponível em: <http://sunsite.berkeley. edu/>. Provê informações sobre coleções e serviços digitais, especialmente sobre direito autoral, metadados, mecanismos de preservação digital, ferramentas e treinamento de recursos humanos.

\section{Livros e manuais}

Uma simples busca na livraria eletrônica Amazon [www.amazon.com], utilizando a expressão "digital library", recupera mais de uma centena de livros sobre o assunto. Este é um tópico que tem atraído crescente atenção por parte dos pesquisadores em variadas áreas - especialmente nas comunidades da ciência da informação e da ciência da computação.

A biblioteca digital é uma temática que muda muito rapidamente. Assim, nenhum livro pode refletir por muito tempo o estado da arte desse assunto. As obras a seguir mencionadas servem, portanto, como marco inicial para compreender os problemas relacionados com as diversas facetas da biblioteca digital. É óbvio que, para conteúdos mais atualizados, devem ser buscadas informações nos trabalhos apresentados em eventos e nos artigos de periódicos.

ANDREWS, Judith; LAW, Derek (Ed.). Digital libraries: policy, planning and practice. Aldershot: Ashgate, 2004, 203 p. Inclui capítulos preparados por 23 autores de vários países, sendo uma obra didática para aqueles que procuram subsídios sobre a política e o planejamento da biblioteca digital. Compreende inúmeros exemplos de projetos de bibliotecas digitais.

ARMS, William. Digital libraries. Cambridge: MIT Press, 2000. Manual clássico e introdutório com conteúdo técnico. Disponível em: <http://www. cs.cornell.edu/wya/DigLib/MS1999/index.html>. A obra está disponível para download de forma gratuita. 
BORGMAN, Christine L. From Gutenberg to the global information infrastructure: access to information in the networked world. Cambridge: MIT Press, 2000, 324 p. Obra introdutória sobre problemas relacionados ao desenvolvimento da biblioteca digital e a chamada infraestrutura global de informação. Nos dois primeiros capítulos são comentados os termos-chave e os principais problemas da temática; o terceiro capítulo traz uma introdução sobre metadados; os capítulos de 4 a 7 cobrem o fluxo da informação, o uso das bibliotecas digitais e o papel das bibliotecas nas instituições; o capítulo 8 comenta a exigência de enfocar as necessidades dos seus conteúdos e na clientela-alvo; no último capítulo a autora aborda a preservação digital e a escalabilidade nos projetos. A obra inclui sumário no final de cada capítulo, um índice e uma bibliografia de 40 páginas.

CHOWDHURY, G. G.; CHOWDHURY, S. Introduction to digital libraries. London: Facet Publishing; New York: Neal Schuman, 2003, 384 p. Obra didática que apresenta uma visão holística do cenário da nova biblioteca digital. Reúne inúmeros exemplos, servindo como fonte para as práticas e técnicas usadas na área. Inclui capítulos sobre definições, projetos, recuperação e avaliação da biblioteca digital.

DAHL, Mark; BANERJEE, Kyle; SPALTI, Michael. Digital libraries: integrating content and systems. Oxford: Chandos, 2006, 203 p. Obra didática em dez capítulos, apresenta as ferramentas e estratégias usadas na implantação de bibliotecas digitais. $\mathrm{O}$ primeiro capítulo fala sobre os requisitos necessários para o projeto da biblioteca digital; os capítulos 2 e 3 apresentam as tecnologias relevantes para as redes e os padrões de XHTML que podem ser utilizados; o capítulo 4 aborda a autenticação e o gerenciamento da identidade e segurança; o capítulo 5 descreve os caminhos pelos quais a biblioteca digital pode se integrar ao sistema de automação da biblioteca; os capítulos 6 e 7 tratam da gestão dos recursos eletrônicos; o capítulo 8 fala das funcionalidades que podem ser utilizadas para a integração com os provedores de conteúdo por meio do OpenURL e metabusca; o capítulo 9 comenta os portais de bibliotecas; o último capítulo aborda as futuras tecnologias que afetarão a biblioteca digital.

HODGES, Patricia et al. (Ed.). Digital libraries: a vision for the 21 st century: a festschrift in honor of Wendy Lougee on the occasion of her departure from the University of Michigan. Ann Arbor: University of Michigan University Library, Scholarly Publishing Office, 2003. Disponível em: < http:// hdl.handle.net/2027/spo.bbv9812.0001.001>. Obra coletiva com oito capítulos que trata da construção da biblioteca digital, do desenvolvimento de coleções e dos aspectos gerenciais do projeto numa biblioteca universitária.

KRESH, Diane (Ed.). The whole digital library bandbook. Chicago: American Library Association, 2007. 416 p. Obra coletiva que aborda, de forma enciclopédica, oito aspectos relacionados com a biblioteca digital: 1) definições: os principais termos e conceitos são definidos sob a forma de pequenos artigos; 2) usuários: os artigos descrevem quem, por que e como as pessoas interagem com a biblioteca digital; 3) o cenário: artigos que descrevem os diversos problemas envolvidos com as bibliotecas digitais; 4) o mercado: artigos que comentam o comércio do conteúdo digital; 5) as ferramentas: locais são comentadas, p. ex., blogs; 6) operações: os artigos descrevem como os bibliotecários colocam a biblioteca digital para funcionar; 7) artigos sobre a preservação digital; 8) o futuro da biblioteca digital.

LESK, Michael. Understanding digital libraries. 2. ed. San Francisco: Morgan Kaufmann, 2004, 456 p. Obra clássica que apresenta uma introdução sobre os desenvolvimentos ocorridos com a biblioteca digital. É dividida em duas partes com 14 capítulos. A primeira se concentra na tecnologia e examina problemas relacionados aos requisitos da mídia, indexação e classificação, redes, distribuição e apresentação dos objetos digitais. A segunda parte aborda os contextos humanos nos quais as bibliotecas digitais operam. Nota: o arquivo da primeira edição, publicada em 2000, está disponível para cópia [URL: http:/ / www.cs.cornell.edu/wya/ DigLib/index.html]. 
MARCONDES, $\mathrm{C}$ et al. (Orgs.). Bibliotecas digitais: saberes e práticas. 2. ed. Brasília: Ibict, 2006. 336 p. Obra coletiva com 16 capítulos que abordam seis aspectos da biblioteca digital: dimensão contextual, dimensão tecnológica, dimensões do uso, gestão em bibliotecas digitais, experiências brasileiras e internacionais. $\mathrm{O}$ foco se dirige a projetos brasileiros em andamento.

MARCUM, Deanna B.; GEORGE, Gerald (Ed.). Digital library development: the view from Kanazawa. Westport: Libraries Unlimited, 2006, 269 p. Com vários ensaios de especialistas de diversos países que abordam a biblioteca digital no meio acadêmico, bem como a influência da tecnologia digital sobre o ensino e aprendizado em geral e o papel da biblioteca nesse contexto educacional.

MARTINEZ EQUIHUA, Saul. Biblioteca digital: conceptos, recursos y estándares. Buenos Aires: Alfragrama, 2007, 230 p. Obra didática introdutória com quatro capítulos que abordam conceitos básicos sobre os diversos tipos de bibliotecas relacionadas ao tema; normas e interoperabilidade; normas computacionais e de conteúdos para as bibliotecas digitais; aplicações de normas em publicações eletrônicas. Inclui exemplos de bibliotecas digitais em países de língua espanhola.

REESE, Terry; BANERJEE, J. Kyle. Building digital libraries: a how-to-do-it manual for librarians. New York: Neal-Schuman, 2008, 277 p. Manual introdutório, de cunho didático, que aborda o planejamento e a avaliação da biblioteca digital; a aquisição, o processamento, a classificação e descrição do conteúdo digital; armazenamento dos documentos; tecnologias utilizadas; compartilhamento dos dados; metabusca; problemas de acesso e uso; migração para novas plataformas e inserção de novos tipos de dados.

ROWLEY, Jennifer; HARTLEY, Richard. Organizing knowledge: an introduction to managing access to information. 4. ed. Guildford: Ashgate, 2008, 392 p. Obra didática, dividida em três partes: 1) comenta a natureza, os conceitos e as práticas para a estruturação e descrição do conhecimento bem como a maneira pela qual os dados, as informações e o conhecimento são integrados dentro das organizações. Além disso trata das ferramentas utilizadas para a organização e recuperação da informação (catálogos, bibliografias, bibliotecas digitais e sistemas de gestão do conteúdo), o armazenamento dos metadados, a estruturação de bases de dados); 2) o acesso à informação: a busca e o comportamento informacional dos usuários, provisão de pontos de acesso, ferramentas para o acesso temático, as classificações bibliográficas e os problemas de catalogação; 3) os diferentes sistemas para a organização do conhecimento: os problemas de gestão desses sistemas e os critérios para a avaliação dos sistemas de gestão do conhecimento.

TAMMARO, Anna Maria; SALARELLI, Alberto. A biblioteca digital. Brasília: Briquet de Lemos, 2008, 378 p. A primeira edição deste livro foi publicada na Itália em 2000; a segunda é de 2006, da qual foi feita a tradução brasileira. É um "manual introdutório às possibilidades e problemas suscitados pelas bibliotecas digitais" (prefácio). Em 13 capítulos, inclui inúmeros exemplos extraídos de projetos localizados em diversos países.

TAYLOR, Arlene G.; JOUDREY, Daniel N. The organization of information. 3. ed. Westport: Libraries Unlimited, 2008, 540 p. Obra clássica que articula a teoria, os princípios, as normas e ferramentas usadas na organização da informação, por exemplo, inventários, bibliografias, catálogos, índices, bases de dados, bem como a evolução histórica da informação registrada. Aborda também as normas de codificação (MARC, SGML), metadados, vocabulário controlado, ontologias e a análise temática dos assuntos.

TEDD, Lucy A.; LARGE, J. Andrew. Digital libraries: principles and practice in a global environment. Munchen: K.G. Saur; 2005, 280 p. Obra didática que aborda em nove capítulos os diversos aspectos da biblioteca digital, a saber: 1) a biblioteca digital e o seu relacionamento com a biblioteca tradicional; 2) os usuários e os serviços; 3) as fontes de informação digital; 
4-6) os aspectos técnicos: padrões e interoperabilidade, organização da informação digital, desenho da interface; 7) busca e acesso: maneiras pelas quais ela pode ser utilizada; 8) operando e gerindo a biblioteca digital; 9) estudo de casos com exemplos de oito bibliotecas da Europa, Ásia e América.

TENNANT, Roy. Managing the digital library. New York: Reed Press, 2004, 280 p. De forma prática, o autor comenta nessa obra os aspectos relacionados com o desenvolvimento de coleções; a catalogação e classificação; a aquisição e digitalização do material; o acesso, os serviços, a organização e os recursos humanos; a tecnologia e infraestrutura, o direito autoral e a preservação. Em cada capítulo foram incluídos links da web que ampliam o conteúdo contido no texto da obra.

THENG, Yin-Leng et al. (Ed.). Design and usability of digital libraries: case studies in Asia Pacific. Hershey, PA: Idea Group, 2009, 690 p. Obra coletiva, que contou com a colaboração de 56 especialistas, dando uma panorâmica dos diversos aspectos da biblioteca digital. Ela inclui 20 capítulos divididos em seis seções: 1) história e cenário do desenvolvimento da biblioteca digital; 2) desenho da arquitetura e dos sistemas; 4) problemas e desafios na implementação; 4) uso e impactos; 5) usuários e usabilidade; 6) tendências futuras da biblioteca digital. A sua ênfase é nos exemplos das práticas de biblioteca digital usadas em instituições dos países asiáticos.

WITTEN, Ian H.; BAINBRIDGE, David. How to build a digital library. San Francisco: Morgan Kaufmann, 2003. Com um conteúdo técnico, explica o uso do programa Greenstone, disponível para download (URL: www.greenstone.org), que pode servir de exercício para aprendizagem ou para a montagem de uma biblioteca digital.

\section{Periódicos especializados}

O assunto biblioteca digital é encontrado em praticamente todos os periódicos da área de ciência da informação. A seguir são comentados somente os títulos específicos.
ARIADNE. Dundee: University of Abertay, University Library, 1996- Trimestral. ISSN 13613200. O seu objetivo é relatar notícias sobre o desenvolvimento de serviços de informação e os problemas das redes de informação. É um periódico eletrônico que inclui artigos e notícias sobre a internet, biblioteca digital e publicação eletrônica. Disponível em: <http://www.ariadne.ac.uk/>.

BULLETIN OF THE IEEE TECHNICAL COMMITTEE DIGITAL LIBRARIES. 2003- Semestral. De cunho técnico, inclui artigos sobre os diferentes aspectos da biblioteca digital. Disponível em: <http://www.ieee-tcdl.org/ Bulletin/current/>.

COMPUTERS IN LIBRARIES. Medford: Information Today, 1988-. ISSN 1041-7915. Título que enfatiza a automação de bibliotecas com avaliações sobre hardware esftware. Disponível em: $<$ http:/ /www. infotoday.com/cilmag/default.shtml $>$.

CURRENT CITES. Berkeley: University of California, Library Technology Watch Program, 1990-. ISSN 1060-2356. Periódico eletrônico, publicado desde 1990, que inclui bibliografia anotada de artigos de periódicos, livros e documentos eletrônicos sobre publicação eletrônica, multimídia, hipermídia, redes e bibliotecas digitais. Disponível em: <http://sunsite.berkeley.edu/CurrentCites/>.

D-LIB MAGAZINE. Reston, VA: Corporation for National Research Institute (CNRI), July 1995 -. ISSN 1082-9873. Título clássico que inclui artigos, notícias e calendário de eventos relacionados com todos os aspectos da biblioteca digital. Disponível em: <www.dlib.org>.

DIGINEWS. Dublin: OCLC, 1979-. ISSN 10935371. Título publicado no período de 1997 a 2007. Entretanto, os artigos podem ser consultados no sítio do OCLC. Ele continha artigos e notícias sobre a prática e tecnologia para a digitalização e preservação digital, bem como análise dos projetos em andamento. Disponível em: < http://www.oclc.org/programs/ publications/newsletters/diginews.htm>. 
DIGITAL DOCUMENT QUARTELY. Saratoga, CA: HGM Consulting, 2001-. Trimestral. ISSN: 1547-8610. Inclui notícias e resenhas de livros/ artigos sobre documentos digitais.

DIGITAL PRESERVATION NEWS. Washington, DC: Library of Congress. Boletim mensal publicado pela Library of Congress que inclui noticiário sobre preservação digital; permite acompanhar as novidades sobre projetos, normas e programas. Disponível em: <http://www.digitalpreservation.gov/ news/archive.html>.ELETRONIC LIBRARY: the international journal for the application of technology in information environments. Oxford: Emerald, Jan. 1983-. ISSN: 0264-0473. Com ênfase em aplicativos e equipamentos utilizados em novas tecnologias, automação de bibliotecas, digitalização, interfaces de usuários e redes relacionadas com todos os tipos de bibliotecas, centros de informação e museus.

\section{INFORMATION TECHONOLOGY AND} LIBRARIES. Chicago: American Library Association, 1982-. Trimestral. ISSN: 0730-9295. Título que publica artigos sobre todos os aspectos relacionados com a tecnologia de informação, incluindo biblioteca digital, metadados, periódico eletrônico, publicação eletrônica, normas, automação de bibliotecas.

INTERNATIONALJOURNAL OF METADATA, SEMANTICS AND ONTOLOGIES. Geneve: Inderscience, 2006-. Trimestral. ISSN: 1744-263X (em linha) e 1744-2621 (impresso). Publica artigos sobre pesquisas e discussões relacionadas com metadados, web semântica e on tologias.

INTERNATIONAL JOURNAL ON DIGITAL LIBRARIES. Berlin: Springer Verlag, 1997-. Trimestral. ISSN 1432-5012 (impresso) e 14321300 (em linha). Primeiro periódico sob a forma impressa, específico sobre biblioteca digital, que tem por objetivo "servir de veículo de disseminação de artigos de pesquisa de alta qualidade que tratem das questões relacionadas com a produção da informação digital, gerenciamento e uso, redes de alta velocidade e conectividade, interoperabilidade e integração de sistemas, pessoas, perfis, necessidades, segurança e privacidade dos indivíduos e das transações comerciais e o processo efetivo do comércio na Idade da Informação" (introdução, v. 1, p. 2). URL: http:/ / link.springer.de [acesso gratuito para quem assina a versão impressa]

INTERNET REFERENCE SERVICES QUARTELY. Philadelphia: Taylor \& Francis, 1996 -. Trimestral. ISSN: 1087-5301. Periódico impresso e eletrônico que inclui artigos e notícias sobre tecnologias e recursos informacionais da Internet.

JOURNAL OF DIGITAL INFORMATION. Texas A\&M University, 1997-. ISSN: 1368-7506. Publica artigos sobre gestão, apresentação e usos da informação em ambientes digitais, incluindo temas como desenho da informação digital, biblioteca digital, hipermídia, gestão da informação, agentes inteligentes, interfaces e consequências sociais da informação digital. Disponível em: <http:// journals.tdl.org/jodi>.

JOURNAL OF ELECTRONIC PUBLISHING. Ann Arbor: University of Michigan. Periódico eletrônico que inclui artigos sobre bibliotecas digitais e arquivos abertos. Enfatiza a publicação eletrônica. Disponível em: <http://www. journalofelectronicpublishing.org/ $>$.

J O UR N A O F I N F ORMATION ARCHITECTURE. Information Architecture Institute, 2009-. Quadrimestral. Periódico eletrônico que tem por objetivo publicar artigos teóricos e práticos sobre os diversos aspectos relacionados com a arquitetura da informação. Disponível em: $<$ http://journalofia.org/>.

JOURNALOFLIBRARY METADATA. Philadelphia: Taylor \& Francis, 1996 -. Trimestral. ISSN: $1937-$ 5034 (eletrônico) 1938-6389 (impresso). Periódico que inclui artigos sobre metadados, catalogação, classificação, ontologias, acesso e organização da informação digital. Nota: durante 1996 e 2006, foi publicado como Journal of internet cataloging.

JOURNAL OF WEB SEMANTICS: science, services and agents on the world wide web. Elsevier, 
2003-. Trimestral. ISSN 1570-8268. Periódico que aborda os diferentes ângulos da produção e impressão de artigos, bem como as ontologias, tecnologias do conhecimento, agentes, bases de dados e redes semânticas. Procura facilitar o desenvolvimento da web semântica, publicando ontologias e seus códigos abertos além de artigos e comunicações. Disponível em: <http://www.informatik.uni-trier. $\mathrm{de} / \sim$ ley/db/journals/ws/index.html $>$.

LIBRARY HI TECH. Emerald, 1983-, Trimestral. ISSN: 0737-8831. Fonte para informações correntes sobre automação de bibliotecas, contendo também artigos sobre bibliotecas digitais.

WORLD DIGITAL LIBRARIES. New Delhi: The Energy and Resources Institute, 2008-. Semestral. ISSN: 0974 - 567X. Periódico internacional, editado na Índia, que publica artigos com enfoque teórico sobre resultados de pesquisa, estudo de casos, gestão e disseminação da informação digital.

\section{Eventos específicos}

A temática de bibliotecas digitais começou a ser discutida em eventos profissionais de cunho geral, notadamente nas áreas de ciência da informação e computação. Com o desenvolvimento do tema, eventos específicos sobre bibliotecas digitais surgiram, sendo que muitos deles já promoveram diversos encontros, inclusive no Brasil. Outras informações sobre os eventos podem ser encontradas no Dlib magazine [URL: www.dlib. org/], em sua coluna "Meetings, Conferences, Workshops". A seguir são mencionados os eventos promovidos de forma regular.

- ACM International Conference on Digital Libraries $₫$ Evento promovido pela Association for Computing Machinery (ACM). Anual.

$1^{\text {st) }}$ March, 20 a 23, 1996, Bethesda, Maryland (US). Proceedings. New York: ACM, 1996. URL: $\mid$ Anais da conferência patrocinada pela Association for Computing Machinery (ACM).

$\left.2^{\text {nd }}\right)$ July, 23 a 26, 1997, Philadelphia, PA. Proceedings. New York: Association for Computing Machinery,
1997. URL: http://www.sigmod.org/dblp/db/ conf/dl/dl97.html

$\left.3^{\text {rd }}\right)$ Digital Libraries '98: Third ACM Conference on Digital Libraries. June, 24 a 27, 1998: Pittsburgh, PA. URL: http://www.sigmod.org/dblp/db/conf/ $\mathrm{dl} / \mathrm{dl} 98 . h \mathrm{html}$

$4^{\text {th }}$ ) Digital Libraries '99: Fourth ACM Conference on Digital Libraries. August, 11 a 14, 1999: University of California, Berkeley. URL: http://www.sigmod. org/dblp/db/conf/dl/dl99.html

$\left.5^{\text {th }}\right)$ Digital Libraries'2000: Fifth ACM Conference on Digital Libraries: June 2 a 7, 2000: San Antonio, TX. URL: http:/ /www.sigmod.org/dblp/db/conf/ $\mathrm{dl} / \mathrm{dl} 2000 . \mathrm{html}$

- Bielefeld International Conference. Bienal. I Evento anteriormente denominado Europäisches Bielefeld Kolloquium oferece, a cada dois anos, discussões estratégicas para administradores de bibliotecas, entre elas, as digitais. É promovido pela Universidade de Bielefeld, Alemanha. Os trabalhos apresentados a partir de 1996, podem ser acessados no URL: http://conference.ub.uni-bielefeld.de/archiv/

$\left.1^{\text {st }}\right)$ Internationales Kolloquium in Bielefeld, February, 18 a 19, 1992.

$\left.2^{\text {nd }}\right)$ Conference Kolloquium, February, 8 a 9, 1994.

$\left.3^{\text {rd }}\right)$ Conference, February, 5 a 7, 1996.

$\left.4^{\text {th }}\right)$ Europäisches Bielefeld Kolloquium, February, 10 a 12, 1998.

$\left.5^{\text {th }}\right)$ Bielefeld 2000 Conference, February, 8 a 10, 2000 .

$\left.6^{\text {th }}\right)$ Bielefeld 2002 Conference, February, 5 a 7, 2002.

$\left.7^{\text {th }}\right)$ Bielefeld International Conference, February, 3 a 5, 2004.

$\left.8^{\text {th }}\right)$ International Bielefeld Conference, February, 7-9, 2006.

$\left.9^{\text {th }}\right)$ International Bielefeld Conference, February 23 a 25, 2009.

- Conferência Ibero-Americana de Publicações Eletrônicas no Contexto da Comunicação 
(CIPECC). Bienal. $\mid$ Evento que tem sua temática e atividades relacionadas à International Conference on Electronic Publishing (ELPUB), e foi idealizado como sua vertente ibero-americana. Por sua abrangência geográfica, é fator de integração entre pesquisadores brasileiros, da região e da comunidade internacional, abrindo espaço para discussão entre diferentes atores do sistema de comunicação científica com distintos projetos e experiências, permitindo o intercâmbio de conhecimentos e a participação em pesquisas coletivas e interdisciplinares.

$1^{\text {a) }}$ Brasília, DF, de 25 a 28 de abril de 2006. URL: http://portal.cid.unb.br/CIPECCbr/papers.php

$\left.2^{a}\right)$ 2008, Rio de Janeiro, 17 de novembro de 2008. URL: http://cipecc2008.ibict.br/index.php/ CIPECC2008/cipecc2008

- DELOS. Anual. $\mid$ Evento promovido pela DELOS Network, vinculada à Comunidade Europeia, que tem por objetivo divulgar o conhecimento das tecnologias da biblioteca digital e suas inúmeras aplicações.

1996, DELOS Workshop, March, 4 a 6, 1996: INRIA - Sophia Antipolis. www.ercim.org/publication/wsproceedings/DELOS1/index.html

1997, DELOS Workshop, November, 10 a 12, 1997: Budapest, Hungary. URL: www.ercim.org/ publication/ws-proceedings/DELOS5/index.html

1998, DELOS Workshop, June, 17 a 19, 1998: Tomar, Portugal. URL: www.ercim.org/publication/ ws-proceedings/DELOS6/index.html

1998, DELOS Workshop, October, 21 a 23, 1998: Stockholm, Sweden. www.ercim.org/publication/ ws-proceedings/DELOS8/index.html

1999, DELOS Workshop, April, 15 a 17, 1999: Brno, Czech Republic. URL: www.ercim.org/publication/ ws-proceedings/DELOS9/index.html

2000, DELOS Workshop, December, 11 a 12, 2000, Zurich, Switzerland. Proceedings. URL: www.ercim. org/publication/ws-proceedings/DelNoe01/

2001, DELOS Workshop, June, 18 a 20, 2001, Dublin City University, Ireland. Proceedings. URL: www.ercim.org/publication/ws-proceedings/ DelNoe02/index.html

2002, DELOS Workshop, June, 6 a 7, 2002, Budapest, Hungary. URL: www.sztaki.hu/conferences/deval/

2003, Delos Workshop, April, 3 a 5, 2003, Helsinki, Finland. URL: http:/ /archivesatrisk.org/aboutfiat/ news/old/2003/2003-02/fiat_iasa_delos_wshop. light.html

2004, DELOS Workshop, October, 4 a 5, 2004, Padua, Italy. URL: dlib.ionio.gr/wp7/ workshop2004_program.html

2005, DELOS Workshop, Heraklion, Crete. May, 11 a 13, 2005. URL: http://www.ukoln.ac.uk/events/ delos-rep-workshop/

2007, DELOS Workshop, June 8, 2007, Budapest, Hungary. URL: www.delos.info/index. php?option $=$ com_content\&task $=$ view\&id $=597 \&$ Itemid $=328$

2008, DELOS Workshop, September 18, 2008, Aarhus, Denmark. URL: http://www.delos.info/ DLFoundations 2008

- Dublin Core and Metadata Initiative (DC). Anual. - É um evento que delibera sobre as normas e os padrões, bem como os esforços para a disseminação da utilização dos metadados.

1995, Dublin, Ohio (US), March, 1 a 3, 1995. URL: http://dublincore.org/workshops/dc1/

1996a, University of Warwick, UK, April, 1 a 3, 1996. URL: http://dublincore.org/workshops/dc2/

1996b, Dublin, Ohio (US), September, 24 a 251996. URL: http://dublincore.org/workshops/dc3/

1997, Helsinki, Finland, October, 6 a 8, 1997. URL: http://www.lib.helsinki.fi/meta/DC5.html

1998, Library of Congress, Washington, DC., USA, November, 2 a 4, 1998. URL: http://dublincore. org/workshops/dc6/

1999, Frankfurt, Germany, October, 25 a 27, 1999.

URL: http://dublincore.org/archives/1999/dc7/ index.htm 
2001, Tokyo, Japan, October, 22 a 26, 2001. URL: http://www.nii.ac.jp/dc2001/

2002, Florence, Italy, October, 13 a 17, 2002. URL: http://www.bncf.net/dc2002/

2003, Seatle (WA), US, September, 28 - October 2, 2003. URL: http://dc2003.ischool.washington. edu/index.html

2004, Shangai, China, October, 11 a 14, 2004. URL: http://dc2004.library.sh.cn/

2005, Madrid, Spain, September, 12 a 15, 2005. URL: http://dc2005.uc3m.es/

2006, Manzanillo, Mexico, October, 3 a 6, 2006. URL: http://dc2006.ucol.mx/

2007, Singapore, August, 27 a 31, 2007. URL: http://www.dc2007.sg/

2008, Berlin, Germany, October, 12 a 16, 2008. Proceedings. URL: http://webdoc.sub.gwdg.de/ univerlag/2008/DC_proceedings.pdf

2009, Seoul, Korea, October, 12 a 16, 2009. URL: www.dc2009.kr/

- Electronic Publishing Conference. Anual. Evento internacional sobre publicação eletrônica que reúne especialistas de diversas áreas, e no que são discutidos aspectos humanos, econômicos, sociais, tecnológicos, legais e comerciais. Os trabalhos apresentados desde 1997 podem ser consultados na ELPUB Digital Repository [URL: http:// elpub.scix.net/cgi-bin/works/Home]. Já foram realizadas:

ELPUB 1997, Canterbury, Reino Unido, de 14 a 16 de abril.

ELPUB 1998, Budapest, Hungria, de 20 a 22 de abril.

ELPUB 1999, Ronneby, Suécia, 10-12 de maio.

ELPUB 2000, Kaliningrad, Rússia, de 17 a 19 de maio.

ELPUB 2002, Karlovy Vary, República Checa, de 6 a 8 de novembro.
ELPUB 2003, Guimarães, Portugal, de 25 a 28 de junho.

ELPUB 2004, Brasília, Brasil, de 23 a 26 de junho. ELPUB 2005, Heverlee, Bélgica, de 8 a 10 de junho.

ELPUB 2006, Bansko, Bulgária, de 14 a 16 de junho. ELPUB 2007, Viena, Áustria, de 13 a 15 de junho.

ELPUB 2008, Toronto Canadá, de 25 a 27 de junho. ELPUB 2009, Milão (Itália), de 10 a 12 de junho.

- Encontro Brasileiro de Arquitetura da Informação (EBAI). Anual. ๆ Evento pioneiro no Brasil focado na arquitetura da informação. Já foram realizados os seguintes:

1) São Paulo (SP), 19 e 20 de outubro de 2007.

2) São Paulo (SP), 17 e 18 de outubro de 2008. URL: http://www.congressoebai.org/index.php/ EBAI/2008/schedConf/program

3) São Paulo (SP), 2 e 3 de outubro de 2009. URL: http://www.congressoebai.org/index.php/ EBAI/2009

- Euro Information Architecture Summit (EUROIA). Anual. II Evento europeu sobre arquitetura da informação (URL: http://www. euroia.org/). Ele é organizado pelo Capítulo Europeu da American Society of Information Science and Technology (ASIST). Já foram realizados os seguintes:

$1^{\text {st) }}$ Bruxelas (Bélgica), 15 e 16 de outubro de 2005.

$\left.2^{\text {nd }}\right)$ Berlim (Alemanha), 30 de setembro-1 de outubro de 2006.

$3^{\text {rd }}$ ) Barcelona (Espanha), 21 e 22 de setembro de 2007.

$\left.4^{\text {th }}\right)$ Amsterdam (Holanda), 26 e 27 de setembro de 2008.

- European Conference on Research and Advanced Technology for Digital Libraries (ECDL). Anual. 9 É considerado o mais importante evento europeu especializado na biblioteca digital e em seus 
problemas técnicos, práticos e sociais. Eventos já realizados:

$1^{\text {st) }}$ Pisa (Itália), September, de 1 a 3, 1997. Proceedings. Berlin: Springer, 1977. ISBN: 3540635548

$\left.2^{\text {nd }}\right)$ Heraklion (Greece), September, de 21 a 23, 1998. Proceedings. Berlin: Springer, 1998. 911 p. ISBN: 3-540-65101-2

$\left.3^{\text {rd }}\right)$ Paris (France), September, de 22 a 24, 1999. Proceedings. Berlin: Springer, 1999. ISBN: 3540665587

$4^{\text {th }}$ ) Lisboa (Portugal), September, de 18 a 20, 2000. Proceedings. Berlin: Springer, 2000. ISBN: 9783540420232 URL: http://www.springerlink. com/content/r6uu0gpfnqbt/

$\left.5^{\text {th }}\right)$ Darmstadt (Germany), September, de 4 a 8, 2001. Proceedings. Berlin: Springer, 2001. 462 p. ISBN: 9783-540425373

$\left.6^{\text {th }}\right)$ Rome (Italia), September, de 16 a 18, 2002. Proceedings. Berlin: Springer, 2002. 664 p. ISBN: 3-540-44178-6

$\left.7^{\text {th }}\right)$ Trondheim, Norway, August, de 17 a 22, 2003. Proceedings. Berlin: Springer, 2003. 536 p. ISBN: 978-3-540-40726-3

$\left.8^{\text {th }}\right)$ Bath (UK), September, de 12 a 17, 2004. Proceedings. Berlin: Springer, 2004. 528 p. ISBN: 3-540-23013-0

$\left.9^{\text {th }}\right)$ Vienna (Austria), September, de 18 a 23, 2005. Proceedings. Berlin: Springer, 2005. 545 p. ISBN: 978354028767-4

$\left.10^{\text {th }}\right)$ Alicante (Espanha), September, de 17 a 22, 2006. Proceedings. Berlin: Springer, 2006. 569p. ISBN: 3-540-44636-2

$11^{\text {th }}$ ) Budapest (Hungria), September, de 16 a 21, 2007. Proceedings. Berlin: Springer, 2007. 585 p. ISBN: 9783540748502

$\left.12^{\text {th }}\right)$ 2008: Aarhus (Dinamarca), September, de 14 a 19, 2008. Proceedings. Berlin: Springer, 2008. 457 p. ISBN: 978-3-540-87598-7 $13^{\text {th }}$ ) Corfu (Grécia), de 27 September a 2 October 2009. URL: http://www.ecdl2009.eu

- Information Architecture Summit (IASUMMIT). Anual. $\mid$ Evento promovido pela American Society of Information Science and Technology (ASIST). Já foram realizados os seguintes:

$1^{\text {st) }}$ Boston (MA, Estados Unidos), 7 a 9 de abril de 2000. URL: www.asis.org/Conferences/ Summit2000/Information_Architecture/index. html

$\left.2^{\text {nd }}\right)$ San Francisco (CA, Estados Unidos), 2 a 4 de fevereiro de 2001. URL: www.asis.org/Conferences/ SUMMITFINAL/Index.html

$\left.3^{\text {rd }}\right)$ Baltimore (MD, Estados Unidos), 15 a 17 de março de 2002. URL: www.iasummit.org/2002/

$4^{\text {th }}$ ) Portland (OR, Estados Unidos), 23 a 27 de março de 2003. URL: www.iasummit.org/2003/

$5^{\text {th }}$ ) Austin (TX, Estados Unidos), 27 a 29 de março de 2004. URL: www.iasummit.org/2004/

$\left.6^{\text {th }}\right)$ Montreal (Canadá), 3 a 7 de março de 2005. URL: www.iasummit.org/2005/

$\left.7^{\text {th }}\right)$ Vancouver (Canadá), 23 a 27 de março de 2006. URL: www.iasummit.org/2006/

$\left.8^{\text {th }}\right)$ Las Vegas (NV, Estados Unidos), 22 a 26 de março de 2007. URL: www.iasummit.org/2007/

$9^{\text {th }}$ ) Miami (FL, Estados Unidos), 10 a 14 de abril de 2008. URL: www.iasummit.org/2008/about.html

- International Conference on Asia-Pacific Digital Libraries (ICADL). Anual. I Evento onde são focalizados os vários aspectos da pesquisa e prática da biblioteca digital. Os anais de 1998 a 2010 podem ser acessados no URL: http://www.icadl.org/

$\left.1^{\text {st }}\right) 1998$ ICADL, Hong Kong (China), 6 e 7 August 1998.

$\left.2^{\text {nd }}\right) 1999$ ICADL, Taipei, Taiwan, November 8 e 9, 1999 .

$3^{\text {rd }}$ ) ICADL 2000, Seoul, Korea, December 10 a 12, 2000. 
$\left.4^{\text {th }}\right)$ ICADL 2001 Mysore, India, December 10 a 12, 2001.

$\left.5^{\text {th }}\right)$ ICADL 2002, Singapore, December 11 a 14, 2002. URL: Proceedings. Berlin: Springer, 2002. 535 p. ISBN 978-3-540-00261-1 URL: http://www. springerlink.com/content/bl8xbrdk813h/

$\left.6^{\text {th }}\right)$ ICADL 2003, Kuala Lumpur, Malaysia, 8 a 12 December, 2003. Proceedings. Berlin: Springer, 2003. 703 p. ISBN 978-3-540-20608-8

$\left.7^{\text {th }}\right)$ ICADL 2004, Shangai, China, December 12 a 17, 2004. URL: http://www.icadl.org/2004/ Proceedings. Berlin: Springer, 2005. 690 p. ISBN 978-3-540-24030-3

$\left.8^{\text {th }}\right)$ ICADL 2005, Bangkok, Thailand, December 10 a 13, 2005. Proceedings. Berlin, Springer, 2005. 529 p. ISBN: 3-540-30850-4

$\left.9^{\text {th }}\right)$ ICADL 2006, Kyoto, Japan, November, 2006.

$\left.10^{\text {th }}\right)$ ICADL 2007, Hanoi, Vietnam, December 10 a 132007.

$\left.11^{\text {th }}\right)$ ICADL 2008, Bali, Indonesia, December 2 a 52008.

12 $\left.{ }^{\text {th }}\right)$ ICADL 2010, Gold Coast, Australia, June 2010.

- International Digital Curation Conference. Anual.

II Evento organizado pelo UK Digital Curation Centre (DCC), Institute of Museum and Library Services (IMLS) e a School of Information and Library Science at the University of North Carolina at Chapel Hill. Ele focaliza os diversos aspectos da preservação digital [URL: http://www.dcc.ac.uk/ events/conferences].

$1^{\text {st) }}$ September, 29 a 30, Bath, England.

$\left.2^{\text {nd) }}\right)$ November, 21 e 22, 2006. Glasgow, Scotland.

$3^{\text {rd }}$ ) December, 11 a 13, 2007, Washington DC, USA.

$\left.4^{\text {th }}\right)$ December, 1 a 3, 2008, Edinburgh (UK).

$\left.5^{\text {th }}\right)$ April, 1 a 3, 2009, Chapel-Hill (NC). URL: http://ils.unc.edu/digccurr2009/

- Joint Conference on Digital Libraries (JCDL). Anual. II Evento conjunto promovido pela
Association of Computer Machinery e o IEEE, por meio do seu IEEE Technical Committee on Digital Libraries (TCDL). Focaliza a biblioteca digital como nova forma de instituições de informação, sistemas operacionais que tratam do conteúdo digital, preservação digital, modelos teóricos de mídia informacional e publicações eletrônicas. É considerada a mais importante nos Estados Unidos. Os anais podem ser acessados no URL: http://www.jcdl.org/past-event-conf.shtml

1'st) JCDL 2001: ACM+IEEE Joint Conference on Digital Libraries, June, 24 a 28, 2001, Roanoke, Virginia, USA.

$\left.2^{\text {nd }}\right)$ JCDL 2002: ACM+Others Joint Conference on Digital Libraries, July, 14 a 18, 2002, Portland, Oregon, USA.

$3^{\text {rd }}$ ) JCDL 2003, Houston, Texas, May, 27 a 312003.

$\left.4^{\text {th }}\right)$ JCDL 2004, Tucson, Arizona, June, 7 a 11, 2004.

$\left.5^{\text {th }}\right)$ JCDL 2005, Denver, Colorado, June, 7 a 11, 2005.

$\left.6^{\text {th }}\right)$ JCDL 2006, Chapel Hill, North Carolina, June, 11 a 15, 2006.

$\left.7^{\text {th }}\right)$ JCDL 2007, Vancouver, British Columbia (Canada), June, 18 a 23, 2007.

$\left.8^{\text {th }}\right)$ JCDL 2008, Pittsburgh, PA, June, 16 a 20, 2008.

$\left.9^{\text {th }}\right)$ JCDL 2009, Austin (TX), June, 15 a 192009.

$10^{\text {th }}$ JCDL 2010, Surfer's Paradise, Australia, June, 21 a $25,2010$.

- Jornadas Españolas de Bibliotecas Digitales. Anual. II Evento que congrega os profissionais espanhóis e estrangeiros.

I) Jornadas Españolas de Bibliotecas Digitales, Valladolid, 6 e 7 de noviembre de 2000. URL: http://alarcos.inf-cr.uclm.es/jisbd2001/jbidi.asp

II) Jornadas Españolas de Bibliotecas Digitales, Almagro, 19 e 20 de noviembre de 2001. URL: http:/ / gaia.dcs.fi.uva.es/ jbidi2001/

III) Jornadas Españolas de Bibliotecas Digitales, Madrid, Universidad. Politécnica, 2002.

Ci. Inf., Brasília, DF, v. 39 n. 1, p.88-107, jan./abr., 2010 
- Jornada sobre la Biblioteca Digital Universitaria. Anual. II Evento realizado a partir de 2003, que congrega bibliotecários argentinos e estrangeiros [URL: http://www.amicus.udesa.edu.ar/jornadasjdbu. html]. É organizado pelas bibliotecas da Red de Bibliotecas de Universidades Privadas, Universidad de Buenos Aires, Universidad Nacional de la Patagonia Austral e a Universidad Nacional de Villa María.

1) Jornada sobre la Biblioteca Digital Universitaria, 20 junio 2003.

2) Jornada sobre la Biblioteca Digital Universitaria JBDU 2004, Buenos Aires, 17 e 18 de junio de 2004.

3) Jornada sobre la Biblioteca Digital Universitaria JBDU 2005, Cordoba, Argentina, 27 e 28 de octubre de 2005.

4) Jornada sobre la Biblioteca Digital Universitaria JBDU 2006. Mendoza, 19 e 20 de octubre de 2006.

5) Jornada sobre la Biblioteca Digital Universitaria JBDU 2007, Buenos Aires, 8 e 9 de novembro de 2007.

6) Jornada sobre la Biblioteca Digital Universitaria JBDU 2008, La Plata, 30 e 31 de octubre de 2008. URL: http://jbdu.fahce.unlp.edu.ar/

7) Jornada sobre la Biblioteca Digital Universitaria JBDU 2009, Rosario, noviembre de 2009.

8) Jornada sobre la Biblioteca Digital Universitaria JBDU 2010, Buenos Aires, 4 e 5 noviembro 2010.

- Libraries in Digital Age (LIDA). Anual. ๆ Evento realizado na Croácia, iniciado em 2000, que tem por objetivo discutir as mudanças e o ambiente em mudança para as bibliotecas, sistemas de informação e serviços no mundo digital [URL: http://web. ffos.hr/lida/]. Em cada ano é escolhido um tema central, dividido em duas partes: pesquisa e desenvolvimento; na segunda parte são discutidos os problemas e as práticas.

$1^{\text {st) }}$ LIDA 2000, Dubrovnik, Croatia, May 25-28, 2000 .

$\left.2^{\text {nd }}\right)$ LIDA 2001, Dubrovnik, Croatia, May, 23 a 27, 2001.

Ci. Inf., Brasília, DF, v. 39 n. 1, p.88-107, jan./abr., 2010 $3^{\text {rd) }}$ LIDA 2002, Dubrovnik, Croatia, May, 21 a 26, 2002.

$\left.4^{\text {th }}\right)$ LIDA 2003, Dubrovnik and Mljet, Croatia, May, 26 a 30, 2003.

$\left.5^{\text {th }}\right)$ LIDA 2004, Dubrovnik and Island of Mljet, May, 25 a 29, 2004.

$\left.6^{\text {th }}\right)$ LIDA 2005, Dubrovnik and Mljet, Croatia, 30 May - 3 June 2005.

$\left.7^{\text {th }}\right)$ LIDA 2006, Dubrovnik and Mljet, Croatia, 29 May - 3 June 2006.

$\left.8^{\text {th }}\right)$ LIDA 2007, Dubrovnik, Croatia, and Island Mljet, Pomena, Croatia, 28 May - 2 June 2007.

$\left.9^{\text {th }}\right)$ LIDA 2008, Dubrovnik and Zadar, Croatia, May, 25 a 30, 2008.

$\left.10^{\text {th }}\right)$ LIDA 2009, Dubrovnik and Zadar, Croatia, May, 25 a 30, 2009.

- Libraries Without Walls. Bienal. I Evento organizado pelo Centre for Research in Library and Information Management (CERLIM) da Manchester Metropolitan University [URL: http:/ / www.cerlim. ac.uk/conf/]. Os anais foram publicados pela editora Facet Publishing (URL: http://www. facetpublishing.co.uk/).

$\left.1^{\text {st }}\right)$ Libraries Without Walls 1, 1995, Greece. Proceedings. Manchester: Cerlim, 1997.

$2^{\text {nd) }) ~ L i b r a r i e s ~ w i t h o u t ~ W a l l s ~ 2, ~ S e p t e m b e r, ~} 17$ a 20, 1997.

$\left.3^{\text {rd }}\right)$ Libraries without Walls 3, September, 1999.

$\left.4^{\text {th }}\right)$ Libraries Without Walls 4, September, 14 a 18, 2001, Molyvos, Lesvos, Greece.

$5^{\text {th }}$ ) Libraries without Walls 5, September, 19 a 23, 2003.

$\left.6^{\text {th }}\right)$ Libraries without Walls 6, September, 16 a 20, 2005.

$\left.7^{\text {th }}\right)$ Libraries Without Walls 7, September, 14 a 18, 2007. 
- Open Repositories Conference. Anual. I Evento idealizado pela University of Sydney e pela Australian National University transformouse numa conferência internacional. Focaliza os problemas e as soluções para as diversas plataformas de repositórios abertos (ex: DSpace, Fedora e EPrints).

$1^{\text {st }}$ International Conference on Open Repositories, Sydney, Australia, January 31- February 3, 2006. URL: http://www.apsr.edu.au/Open_ Repositories_2006/

$\left.2^{\text {nd }}\right)$ International Conference on Open Repositories, January 23-26, San Antonio (TX), United States. URL: http://openrepositories.org/2007/

$\left.3^{\text {rd }}\right)$ International Conference on Open Repositories, Southhampton, England, April 4, 2008. URL: http://pubs.or08.ecs.soton.ac.uk/view/subjects

$\left.4^{\text {th }}\right)$ International Conference on Open Repositories, Atlanta (GA), United States, May 18-21, 2009. URL: https://or09.library.gatech.edu/

- Reference Renaissance I No período de 1999 a 2004, o evento era denominado Virtual Desk Conference; sua nova denominação teve início em 2005. Reúne os interessados na referência digital ou on-line, sendo promovido pelo Bibliographical Center for Research e pela Reference and User Services Association (RUSA).

VRD 2000, Virtual Reference Desk Conference October, 2000, Seattle, Washington. URL: www. vrd.org/.

VRD 2001, Virtual Reference Desk Conference, $3^{\text {rd }}$ : 2001: Orlando, Florida. Proceedings. New York: Neal-Schuman, 2003, 232 p. ISBN 1555704506 I Nota: os anais do evento estão dividido em seis partes: 1) identificando as necessidades dos serviços de referência digital que utilizam o correio eletrônico; 2) gerindo os problemas-chave da referência digital: privacidade, direitos autorais, entrega dos resultados; 3) implementando o serviço em tempo real; 4) concebendo e implementando os serviços de referência colaborativos; 5) pesquisa sobre o uso da referência digital; 6) avaliação da referência digital.
VRD 2004, Virtual Reference Desk Conference 2004, 4 ${ }^{\text {th }}$, Cincinati, Ohio, November, 8 e 9, 2004. URL: www.vrd2004.org/

RR 2008, August, 4 e 5, 2008, Denver, Colorado. URL: http://www.bcr.org/referencerenaissance/2008/ index.html

RR 2010, August, 8 a 10, 2010, Denver, Colorado. URL: http://www.bcr.org/referencerenaissance/

- Russian Conference on Digital Libraries (RCDL). Anual. II Evento que congrega pesquisadores e especialistas russos e estrangeiros, focalizando os diversos aspectos da biblioteca digital.

1) 1999, St. Petersburg, Russia, October, 18 a 22. URL: http://www.dl99.nw.ru/

2) 2000, Protvino, Russia.

3) 2001, Petrozavodsk, Russia, September, 11 a 13.

URL: http://rcdl2001.krc.karelia.ru/\#top

4) 2002, Dubna, Russia, October, 15 a 17, 2002. URL: http://rcdl2002.jinr.ru

5) 2003, Saint Petersburg, Russia, October, 29 a 31.

6) 2004, Pushchino, September 29 - October 1, 2004.

7) 2005, Yaroslavl, Russia, October, 04 a 06, 2005. URL: http://www.rcdl2005.uniyar.ac.ru/en/news. shtml

8) 2006, Suzdal, Russia, October, 17 a 19, 2006. URL: http://www.rcdl2006.uniyar.ac.ru/en/news. shtml

9) 2007, Pereslavl, Russia, October, 15 a 18, 2007. URL: http://rcdl2007.pereslavl.ru/en/news.shtml

10) 2008, Dubna, Russia, October, 7 a 11, 2008. URL: http://rcdl2008.jinr.ru/eng/

11) 2009, Petrozavodsk, Russia, September, 11 a 21, 2009. URL: http://rcdl2009.krc.karelia.ru/index. php?plang $=\mathrm{e}$

- Simpósio Internacional de Bibliotecas Digitais. Bienal. I Evento inicialmente denominado Workshop Política de Informação em Bibliotecas

Ci. Inf., Brasília, DF, v. 39 n. 1, p.88-107, jan./abr., 2010 
Digitais, é promovido em parceria entre o Ibero American Science \& Technology Education Consortium (ISTEC), a Universidade de Campinas, a Universidade de São Paulo e a Universidade Federal de São Paulo. Seu objetivo é difundir as pesquisas e as práticas nas bibliotecas digitais.

1) Workshop Política de Informação em Bibliotecas Digitais, 17-21 de março de 2003. URL: http:// libdigi.unicamp.br/

2) Simpósio Internacional de Bibliotecas Digitais, Campinas (SP), 17-21 de maio de 2004. URL: http:/ / libdigi.unicamp.br/document/list.php?tid $=82$ I Acesso aos trabalhos apresentados no evento. - Anais do II Simpósio Internacional de Bibliotecas Digitais. Campinas: Universidade de Campinas, 2004. URL: http://libdigi.unicamp.br/document/list. php?tid $=82$

3) Simpósio Internacional de Bibliotecas Digitais, São Paulo (SP), de 28 de novembro a 2 de dezembro de 2005. I Anais: A dimensão social da Biblioteca Digital na organização e acesso ao conbecimento: aspectos teóricos e aplicados. São Paulo: Universidade de São Paulo, 2005. 2 v. 1100 p.

4) Simpósio Internacional de Bibliotecas Digitais, São Paulo (SP), de 18 a 20 de setembro de 2007. Anais no URL: http://libdigi.unicamp.br/ document $/$ list.php?tid $=375$

- Symposium on Electronic Theses and Dissertations (ETD). Anual. ๆ Evento organizado pelo Networked Digital Library of Theses and Dissertations (NDLTD). Tem por objetivo discutir os problemas e as soluções relacionados com as teses e dissertações eletrônicas. Já foram realizados os seguintes:

ETD 1998, Memphis (TN), Estados Unidos, 10-11 de junho.

ETD 1999, Blacksburg (VA), Estados Unidos, 16 a 18 de maio.

ETD 2000, St. Petersburgh (FL), Estados Unidos.

ETD 2001, Pasadena (CA), Estados Unidos, 22 a 24 de maio. URL: http://www.wvu.edu/ thesis/ News/4th_ETD_Symp_2001_Web_Site.pdf
ETD 2002, Provo (UH), Estados Unidos, de 30 de maio a 1 de junho. URL: http://www.dl-forum.ptdlr.de/englisch/veranstaltungen/29_1045_ENG_ HTML.htm

ETD 2003, Berlin, Alemanha, 21 a 24 de maio. URL: http://www2.hu-berlin.de/etd2003/

ETD 2004, Lexington (KY), Estados Unidos, 3 a 5 de junho. URL: http://www.uky.edu/ETD/ ETD2004/

ETD 2005, Sydney, Austrália, 28 a 30 de setembro. URL: http://adt.caul.edu.au/etd2005/etd2005.html

ETD 2006, Quebec, Canadá, 7 a 10 de junho. URL: http://www6.bibl.ulaval.ca:8080/etd2006/pages/ prog.jsf

ETD 2007, Uppsala, Suécia, 13 a 16 de junho. URL: http://epc.ub.uu.se/ETD2007/

ETD 2008, Aberdeen, Escócia, 4 a 7 de junho. URL: http://www.rgu.ac.uk/etd/home/page.cf $\mathrm{m} ? \mathrm{CFID}=21950889 \& \mathrm{CFTOKEN}=15169155 \&$

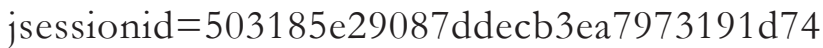
5632a3TR

ETD 2009, Pittsburgh, Estados Unidos, 10 a 13 de junho. URL: http://www.library.pitt.edu/etd2009/

\section{Listas de discussão}

As listas de discussão são importantes para o acompanhamento dos novos projetos, equipamentos e aplicações.

AIFIA-pt. Lista de discussão mantida pelo Information Architecture Institute, dedicada a debater assuntos relacionados ao campo de estudo da arquitetura da informação. Contato: aifia-pt@ lists.ibiblio.org

BIBLIOTECAS e Tecnologias da Informação e Comunicação (bib_virtual). Disponível em: $<$ http://listas.ibict.br/cgi-bin/mailman/listinfo/ bib_virtual>.E-mail: bib_virtual@ibict.br. Lista de discussão sobre bibliotecas digitais criada em 1996 pelo Ibict. 
CONSERVATION DistList. Disponível em: $<$ http://cool-palimpsest.stanford.edu/byform/ mailing-lists/cdl/>. Lista iniciada em 1988, gerenciada pelo Conservation Lab Stanford University Libraries, que cobre a conservação e preservação da informação digital.

DIGITAL Copyright. Disponível em: < http:// www.umuc.edu/distance/odell/cip/listserv. shtml\#digital>. Lista mantida pelo Center for Intellectual Property da University of Maryland, tem por objetivo a discussão do desenvolvimento das aplicações das leis do direito autoral no ambiente on-line.

DIGITAL-preservation. Disponível em: < https:/ / www.jiscmail.ac.uk/cgi-bin/webadmin? A0= digitalpreservation $>$. Lista criada em 2000, gerenciada pelo UK Joint Information Systems Committee, tem por objetivo a discussão dos projetos e as atividades da preservação digital.

DIGLIB. IFLA Digital Libraries Research. E-mail: diglib@infoserv.inist.fr. Lista de discussão sobre bibliotecas digitais, hospedada no Institute de l'Information Scientifique et Technique (INIST, França) e gerenciada pela IFLA. Teve início em 1995 e é moderada por Terry Kuny.

IMAGELIB. Disponível em: < http:/ listservarizona. edu/archives/imagelib.html>. Criada em 1994, é gerenciada pela University of Arizona Library. Discute os assuntos ligados aos projetos de imagem digital.

INTEROPERABILITY Focus Discussion List. Disponível em: <http://www.jiscmail.ac.uk/lists/ interoperability.html $>$. Lista de discussão gerenciada pelo UK Office for Library and Information Networking (UKOL), que focaliza os problemas relacionados com o desenho, a construção e operação de serviços interoperáveis num ambiente digital.

JISC Electronic Libraries Research Programme. Disponível em: <www.ukoln.ac.uk/services/elib/ mailing-lists/lis-elib.html>. Lista de discussão sobre os projetos de bibliotecas digitais no Reino Unido.
LIS-ELIB. Disponível em: <https://www. jiscmail.ac.uk/cgi-bin/webadmin?SUBED1=LISELIB\&A=1>. Lista de discussão do JISC Electronic Libraries Programme e seus projetos.

LIBLICENSE-L Mailing List. Disponível em: $<$ http://www.library.yale.edu/ llicense/mailinglist.shtml>. Lista criada em 1999, e mantida pela Yale University Library. Está voltada aos problemas relacionados com o licenciamento da informação digital pelas bibliotecas.

MANAGEMENT \& Preservation of Electronic Records. Disponível em: <https://listservalbany. edu/cgi-bin/wa?A0=ERECS-L>. Iniciada em 1997, é uma lista moderada na qual são discutidas as ideias, técnicas e problemas relacionados com a gestão e preservação dos registros eletrônicos.

OAI-General. Disponível em: <http://www. openarchives.org/mailman/listinfo/OAIgeneral>. Lista da Open Archives Initiative (OAI), centra-se no desenvolvimento e na promoção dos padrões de interoperabilidade, visando a facilitar a eficiente disseminação do conteúdo informacional.

PADIFORUM. Disponível em: <http://listserver. nla.gov.au/wws/info/padiforum-l>. Lista de discussão sobre preservação digital mantida pela National Library of Australia.

RIGHTS-L. Disponível em: <http://listserv.utk. edu/archives/rights-1.html>. Criada em 2001, gerenciada pela University of Tennessee, preocupase com a gestão dos direitos digitais de documentos de todos os gêneros e formatos.

XML4LIB Electronic Discussion. Disponível em: $<$ http://lists.webjunction.org/xml4lib/>. Lista de discussão criada em 2001, debate o XML e sua utilização em bibliotecas.

WEB-ARCHIVE. Disponível em: < http://listes. cru.fr/wws/info/web-archive>. Lista gerenciada pelo Comité Réseau des Universités (França), foca no arquivamento de conteúdos digitais em linha.

Ci. Inf., Brasília, DF, v. 39 n. 1, p.88-107, jan./abr., 2010 
WEB Curators Mailing List. Disponível em: $<$ http://netpreserve.org/about/curator.php $>$. Lista mantida pelo International Internet Preservation Consortium, indicada para curadores da web e outros especialistas interessados na busca na internet, qualidade e problemas de arquivamento.

WEB4LIB Electronic Discussion. Disponível em: $<$ http://lists.webjunction.org/web4lib/>. Criada em 1994, discute os problemas de gestão das bibliotecas hospedadas na web.

ZIG Discussion list. Disponível em: <www-zigrequest@w3.org>. Lista mantida pelo W3C, foca os problemas do Protocolo Z39.50.

\section{Grupos e centros de pesquisa}

Os grupos e centros de pesquisa sobre bibliotecas digitais surgiram em meados dos anos 1990. É certo que a biblioteca digital é motivo de inúmeras pesquisas individuais; no Brasil, por exemplo, tal fato pode ser comprovado em busca na base de dados da Plataforma Lattes [URL: www.cnpq.br], que traz como resultado nomes de dezenas de pesquisadores nas mais diversas áreas científicas e tecnológicas.

A seguir são listados os principais grupos e centros de pesquisa que possuem estrutura formal dentro de suas organizações.

IEEE Digital Library Task Force. Disponível em: <http://cimic.rutgers.edu/ieee_dltf.html>. Grupo de especialistas criado em 1995 pela IEEE Computer Society, para discutir os aspectos técnicos da área de informática relativos à biblioteca virtual.

RUTGERS University. Center for Electronic Texts in the Humanities. Disponível em: <http:// www.ceth.rutgers.edu/ $>$. Dedica-se aos projetos de pesquisa em biblioteca digital nas áreas das humanidades.

TEXAS A \& M University. Center for the Study of Digital Libraries (CSDL). Disponível em: <http:// www.csdl.tamu.edu/>. Criado em 1995, pela Texas A \& M University, tem por objetivo pesquisar os aspectos teóricos e práticos da biblioteca digital. O Centro já organizou duas conferências profissionais.

UNIVERSITY of Massachusetts Amherst. Center for Intelligent Information Retrieval (CIIR). Disponível em: < http:/ / ciir.cs.umass.edu/ membership/member.html>. Centro voltado para a pesquisa nas áreas de recuperação e extração da informação. Já publicou mais de 800 artigos nessas áreas e trabalha com mais de 90 instituições públicas e privadas.

UNIVERSITY of Strathclyde. Centre for Digital Library Research (CDLR). Disponível em: <http:/ / cdlr.strath.ac.uk/>. Criado em 2000, é uma iniciativa conjunta do Information Resources Directorate e o Department of Computer and Information Sciences com o objetivo de combinar a teoria com a prática em modos inovadores e ser um centro de excelência nas áreas de distribuição da informação digital e ambientes de aprendizagem.

VIRGINIA Tech University. Digital Library Research Laboratory. Disponível em: <http:// www.dlib.vt.edu/DLRL_Mission.html >. Pesquisa os aspectos relacionados com a recuperação da informação, especialmente a multimídia e visualização.

\section{Cursos e treinamentos}

Nos últimos anos começaram a aparecer disciplinas voltadas às bibliotecas digitais em inúmeras universidades tanto estrangeiras como nacionais. Além disso, também apareceram cursos especializados sobre a temática.

O Ibict, com o intuito de programar a sua Biblioteca Digital Brasileira de Teses e Dissertações [URL: http://bdtd.ibict.br/], tem promovido inúmeros cursos e treinamentos relacionados com as bibliotecas digitais de teses e dissertações nas instituições de ensino superior. O Instituto também tem ofertado dezenas de cursos de treinamento sobre o Sistema Eletrônico de Editoração de Revistas (SEER) [URL: http://seer. ibict.br/] - um software traduzido e customizado, baseado no sistema desenvolvido pelo Public 
Knowledge Project (Open Journal Systems) da Universidade British Columbia (Canadá).

A seguir estão listados os cursos e treinamentos que têm tido ofertas regulares.

CORNELL University Library. Department of Preservation and Conservation Digital Imaging for Libraries and Archives. Disponível em: <http:// www.library.cornell.edu/preservation/training/ index.html>. Curso com duração de uma semana sobre uso de tecnologia de imagem digital em bibliotecas e arquivos.

TILBURG University (Holanda). International Summer School on the Digital Library. Disponível em: <http://www.tilburguniversity.nl/services/lis/ ticer/>. Cursos sobre biblioteca digital, ofertados a partir de 1996, com duração de duas semanas. Geralmente realizados em agosto.

UNIVERSIDADE Federal do Pará. Instituto de Ciências Sociais Aplicadas. Curso de Especialização em Gestão da Informação em Bibliotecas Digitais. Disponível em: <www3.ufpa.br/multicampi/ images/LINK/bd.pdf > . Curso de especialização ofertado 2008 e 2009, com duração de 14 meses.

UNIVERSITY of Michigan, School of Information. Digital Tool Kit. Disponível em: <http://www. si.umich.edu/>. Cursos rápidos (de 2 a 5 dias) sobre diversos tópicos.

\section{Principais organizações}

Dezenas de organizações estão envolvidas com a biblioteca digital. Abaixo estão listadas aquelas que têm ocupado papéis mais relevantes, entre outros, na implementação de projetos, fomento para projetos, normalização e preservação digital.

AMERICAN Institute for Conservation of Historic and Artistic Works (AIC). Disponível em: $<$ http:/ /aic.stanford.edu > Instituição que congrega organizações e especialistas da área de conservação de obras históricas e artísticas.
ASSOCIATION of Research Libraries. Disponível em: <www.arl.org > . Mantém dois programas de interesse: o Digital Initiatives Database [Disponível em: <http://www.arl.org/did >..] e o Preservation Program [Disponível em: <http://www.arl.org/ preserv/>..].

CANADIAN Initiatives on Digital Libraries. Disponível em: <http://epe.lac-bac.gc.ca/100/206/301/lac-bac/ cidl-ef/2007-09-28/cidl/index-e.html>. Aliança de bibliotecas canadenses que tem por objetivo promover, coordenar e facilitar o desenvolvimento das coleções e serviços digitais canadenses. Publica o CIDL Newss, noticiário bilíngue.

COMUNIDADE Européia. Program of Digital Library and Culture (DIGICULT). Disponível em: $<$ http://cordis.europa.eu/fp7/ict/telearn-digicult/ digicult_en.html>. Programa que provê recursos financeiros para projetos relacionados com a herança cultural, biblioteca digital e preservação digital.

CONSELHO Nacional de Arquivos (CONARQ). Disponível em: <http://www.conarq. arquivonacional.gov.br/cgi/cgilua.exe/sys/start. htm>. Órgão colegiado, vinculado ao Arquivo Nacional, que tem por finalidade definir a Política Nacional de Arquivos Públicos e Privados, como órgão central de um sistema nacional de arquivos, bem como exercer orientação normativa visando à gestão documental e à proteção especial aos documentos de arquivo.

CONSERVAÇÃO Preventiva em Bibliotecas e Arquivos (CPBA). Disponível em: $<$ http://143.106.151.46:80/cpba/>. Projeto brasileiro que tem o objetivo de ampliar o conhecimento à preservação dos acervos documentais por meio de um programa de informação e intercâmbio. Provê acesso a 52 títulos sobre a conservação preventiva de livros e documentos, filmes, fotografias e meios magnéticos. Estes textos abordam o planejamento e o gerenciamento de programas institucionais; o controle das condições ambientais, da prevenção contra riscos e do salvamento de coleções em situações de emergência, 
da armazenagem, conservação e reformatação, envolvendo os recursos da reprodução eletrônica, da microfilmagem e da digitalização.

COUNCIL on Library and Information Resources (CLIR). Disponível em: <http://www.clir.org/>. Tem por objetivo estimular novos enfoques para a gestão da informação digital, de modo que estejam disponíveis no futuro. Publica inúmeros relatórios técnicos.

DELOS Network of Excellence on Digital Libraries. Disponível em: <http://www.delos. info/>. Focaliza na divulgação das teorias da biblioteca digital e no desenvolvimento de serviços informacionais interoperáveis, além de gestão do conteúdo integrado.

DIGITAL Curation Centre. Disponível em: <http://www.dcc.ac.uk/>. Provê a disseminação da pesquisa e solução dos problemas relacionados com o armazenamento, a gestão e a preservação de recursos informacionais.

DIGITAL Library Federation (DLF). Disponível em: <http://www.clir.org/dlf. html>. Consórcio de bibliotecas e instituições ligadas ao uso das tecnologias da informação eletrônica visando a expansão de suas coleções e serviços. Disponibiliza inúmeros relatórios e documentos técnicos sobre a biblioteca digital. Em abril de 2009 integrou-se ao Council on Library and Information Resources.

DIGITAL Preservation Coalition. Disponível em: $<$ http://www.dpconline.org/>. Instituição que tem por objetivo divulgar a preservação do material digital, bem como os seus problemas estratégicos, culturais e tecnológicos.

DSPACE (MIT). Disponível em: < http://www. dspace.org/>. Fundação que disponibiliza o programa para a organização de repositório digital.

DUBLIN Core Metadata Initiative. Disponível em: <http://dublincore.org/>. Trabalha no desenvolvimento de normas para metadados.
EUROPEAN Commission on Preservation and Access. Disponível em: <http://www.knaw.nl/ ecpa/>. Criada em 1994, com sede na Holanda, tem por objetivo promover as atividades de preservação digital das bibliotecas e arquivos da Europa. Publica inúmeros documentos sobre o assunto.

GREENSTONE Digital Library Software. Disponível em: <http://www.greenstone.org/>. Provê a distribuição do pacote de programas Greenstone usado no desenvolvimento de biblioteca digital.

INTERNATIONAL Federation of Library Associations and Institutions (IFLA). Disponível em: <http://www.ifla.org/>. Fundada em 1927, é a associação internacional com membros de mais de 150 países, que representa os interesses da biblioteca, serviços de informação e seus usuários.

INFORMATION Architecture Institute. Disponível em: <http://www.iainstitute.org/>. Organização sem fins lucrativos, com enfoque internacional, que tem por objetivo congregar indivíduos e organizações ligadas ao desenho e construção de ambientes informacionais compartilhados. Publica: Information Architecture Institute (LAI) Newsletter; Journal of Information Architecture.

INSTITUTO Brasileiro de Informação em Ciência e Tecnologia (Ibict). Disponível em: <www.ibict. br>. Organismo nacional que tem por objetivo promover a competência, o desenvolvimento de recursos e a infraestrutura de informação em ciência e tecnologia para a produção, socialização e integração do conhecimento científico-tecnológico. Desenvolve produtos e serviços relacionados com periódico eletrônico, teses e dissertações no formato digital [URL: $<$ http://bdtd.ibict.br/>.], repositórios digitais [URL: $<$ http://dspace.ibict.br/>.] e Incubadora de Revistas Inseer [URL: < http://inseer.ibicINTERNATIONAL Center for the Study of the Preservation and Restoration of Cultural Property. Disponível em: $<$ http://www.iccrom.org/eng/00about_en.shtml>. Organismo intergovernamental, sediado em Roma (Itália), que tem por objetivo promover e difundir a conservação de bens culturais. 
INTERNATIONAL Council of Archives. Committee on Electronic Records. Disponível em: <www.ica.org/>. Instituição internacional que promove a preservação e uso dos arquivos em todo o mundo. No seu Resource Center [http:/ / www.ica.org/en/resource-centre] estão disponíveis documentos e normas relevantes para a prática arquivística.

INTERNET Archive. Disponível em: < http:// www.archive.org/>. Instituição sem fins lucrativos que prove acesso aos sítios da Web. É importante para se recuperar páginas antigas ou que deixaram de estar disponíveis. Coordena também a Open Content Alliance, um esforço colaborativo de organizações culturais e tecnológicas de diversos países, que ajuda na montagem de um arquivo permanente de textos digitalizados e material multimídia.

NATIONAL Library of Australia. Preserving Access to Digital Information (PADI), Australia. Disponível em: < www.nla.gov.au/padi/> Programa da Biblioteca Nacional da Austrália que tem por objetivo prover informações e mecanismos para a preservação digital. Editou uma série de guias e promove eventos regulares sobre o tema.

NETWORKED Digital Library of Theses and Dissertations (NDLTD). Disponível em: < http:// www.ndltd.org/>. Organização internacional dedicada à promoção da adoção, criação, uso, disseminação e preservação das teses e dissertações em suporte digital. Provê acesso a documentos e normas relacionadas com esse tipo de biblioteca digital.

NORTHEAST Document Conservation Center. Disponível em: <www.nedcc.org/>. Instituição norte-americana que tem por objetivo melhorar os esforços de conservação das bibliotecas, arquivos, organizações históricas, museus e outros repositórios, provendo serviços de qualidade para essas instituições. É reconhecida pelos programas educativos e pela promoção da Digital Directions Conference.
ON-LINE Computer Library Center (OCLC). Disponível em: <www.oclc.org/>. Instituição que provê documentos e normas sobre digitalização, catalogação, metadados, preservação digital e referência digital. Sedia o Worldcat, o maior catálogo coletivo com acervos de centenas de bibliotecas, bem como o Research Library Group.

OPEN Archives Initiative (OAI). Disponível em: <www.openarchives.org/>. Instituição que desenvolve e promove as normas de interoperabilidade que têm por objetivo facilitar a disseminação eficiente de conteúdos informacionais. Promove também ações de arquivo aberto e repositórios institucionais.

ORGANIZAÇÃO das Nações Unidas para Educação, Ciência e Cultura (Unesco). Disponível em: <http://www.unesco.org/>. Organismo internacional que provê inúmeras fontes de informação sobre biblioteca digital. São elas:

- Unesco Archives Portal. Disponível em: <www. unesco.org/webworld/portal_archives/pages/ index.shtml>. Inclui seções com links para "Fontes primárias em linha" e "Preservação e conservação: preservação digital”.

- Unesco Library Portal. Disponível em: www. unesco.org/webworld/portal_bib/. Inclui uma seção que apresenta links para "Iniciativas de preservação e acesso".

- Unesco. Memory of the World. Disponível em: $<$ www.unesco.org/webworld/memory/mempage. htm $>$. Programa da Unesco que tem por objetivo proteger e divulgar os documentos importantes para a cultura mundial. No sítio são mostrados inúmeros produtos e projetos.

WORLD Wide Web Consortium (W3C). Disponível em: <http://www.w3.org/>. Organismo que desenvolve as tecnologias de interoperabilidade (especificações, guias, software e ferramentas) com o objetivo de aperfeiçoar o uso da internet. O site do Escritório Brasileiro, inaugurado em 2007, está localizado no: < http://www.w3c.br/>.

Ci. Inf., Brasília, DF, v. 39 n. 1, p.88-107, jan./abr., 2010 


\section{CONSIDERAÇÕES FINAIS}

O assunto biblioteca digital, conforme se pode depreender pela bibliografia apresentada, tem tido crescimento acentuado desde meados dos anos 1990. De três congressos profissionais, especificamente dedicados ao tema em 1994, o número cresceu para cinco em 1995; para oito, em 1997 e, em 2009, já ultrapassava uma dezena. Vários títulos de periódicos específicos - por exemplo, o D-Lib Magazine e o International Journal on Digital Libraries - passaram a divulgar artigos sobre pesquisas e projetos em andamento. Além disso, livros, listas de discussão, artigos de periódicos e teses demonstram que o tema está em pleno crescimento. Inicialmente restrito aos Estados Unidos e Reino Unido, começa a disseminar-se em outros países.

A biblioteca digital é uma área que tem grande futuro na ciência da informação. Assim, esta bibliografia pretendeu colaborar na divulgação do assunto e motivar estudos e pesquisas relativas ao tema.

Artigo submetido em 25/05/2009 e aceito em 08/07/2010. 